\title{
Modelling of Hydrological Drought Events in the Upper Tana Basin of Kenya
}

\author{
Jones F. Agwata ${ }^{1}$, Wellington N. Wamicha ${ }^{2}$, Christopher M. Ondieki ${ }^{3}$ \\ ${ }^{*}$ Centre for Advanced Studies in Environmental Law and Policy, University of Nairobi, PO Box 30197, 00100 \\ GPO Nairobi; \\ ${ }^{2}$ Formerly of Department of Environmental Sciences, Kenyatta University, PO Box 43844, 00100, Nairobi \\ ${ }^{3}$ Department of Geography, Kenyatta University, PO Box 43844, 00100 Nairobi
}

\begin{abstract}
Drought is a recurring hazard which affects many parts of Kenya. In most countries in Sub-Saharan Africa, agriculture which is predominantly rain-fed is the main stay of the economies is highly prone to the impacts of drought which, whenever it occurs, leads to serious socioeconomic challenges at various levels. The study of drought duration, magnitude and severity have relevance in many areas such as waste load allocations, issuance of pollution discharge permits, location of treatment plants and sanitary landfills, determination of allowable water transfers and withdrawals both within, between and outside the affected areas and determination of minimum downstream release requirements for hydropower water supply, cooling plants and other facilities. Knowledge of the frequency distribution of the drought events is useful as it contributes to the assessment of drought risks which have implications on the long term ecological, economic and social well being of the biological and human communities that make use of water from the various streams in a basin. In this study five frequency distributions were fitted to drought duration and severity as determined from discharge data from representative river gauge stations in the upper Tana Basin of Kenya. The frequency distributions fitted to the two drought events were the Generalized Normal $(G N)$ or 3-parameter Lognormal, Generalized Extreme Value (GEV) or the Extreme Value Type III, Generalized Pareto (GPA), Pearson Type III (P3) and Generalized Logistic (GL). The distributions of best fit for the drought events were identified using the $Z$ value obtained from the average L-moment statistics of a particular candidate distribution and the average L-moment statistics. The $Z$ value for each homogenous region was determined from sample estimates of $L_{c v}, L_{c s}$ and $L_{c k}$ that were determined from probability weighted moment estimators and the weighted means of $L_{c v}$, $L_{c s}$ and $L_{c k} u s i n g$ records from the river gauging stations representing each hydrologically homogenous region. A frequency distribution of best fit was selected if $\left|Z^{D i s}\right| \leq 1.64$ and the one with the lowest $\left|Z^{D i s}\right|$ value selected as the distribution of best fit. Results showed that the frequency distribution of best fit for duration and severity was the Generalized Normal while the Pearson Type III distribution was the distribution of worst fit for both duration and severity.

Key words: hydrological drought, drought events, frequency distribution, modeling
\end{abstract}

\section{Introduction}

Drought events modelling is about fitting several frequency distributions to drought duration, severity and magnitude and selecting the distribution of best fit using a given criterion or several criteria. Since stream flow records cannot assume values less than zero, the candidate frequency distributions normally used in droughts events modelling are those that are bounded below (that is, have lower limits equal to or greater than 0 ) and are defined by no more than three parameters. Consequently, frequency distributions with a lower bound and those with three parameters were deemed suitable for use in this study. The distributions include the Generalized Normal (Three Parameter Lognormal), Generalized Extreme Value (Weibull Type III), Pearson Type III and the Generalized Logistic and have previously been widely used in various studies by Vogel and Kroll (1989). To derive the risk of occurrence of the magnitude of a particular drought event, it is necessary to know the frequency distribution that best describes past characteristics of the drought event. This requires that a suitable drought frequency model be determined from a group of competing ones through fitting the available historical hydrological drought events data to various frequency distribution models and selecting, using a suitable criteria, the most appropriate frequency model.

Empirically, drought frequency analysis involves fitting several statistical frequency distributions to the drought events and then selecting the model of best fit for each of the events. The frequency distribution models to be fitted should be those that comply with practical statistical and physical considerations. Since drought events cannot assume values less than zero, it is necessary that candidate distributions be bounded below (that is, have lower limits equal to or greater than 0 ) and be defined by no more than three parameters. Consequently, only those probability distributions with a lower bound and those defined by three parameters are best suited to drought events frequency analysis. Five three-parameter statistical frequency distributions were used to model the drought events in the delineated homogeneous regions of the upper Tana basin. 
The frequency distributions fitted were the Generalized Normal (GN) or 3-parameter Lognormal, Generalized Extreme Value (GEV) or the Extreme Value Type III, Generalized Pareto (GPA), Pearson Type III (P3) and Generalized Logistic (GL). Details of these distributions and their applications are available in Chowdhury et al (1991). The distributions were selected because they are widely applied in drought frequency analysis, are simple and convenient to apply and are consistent, flexible and robust. In addition, they are theoretically well based, are well documented, have lower and upper bounds and are defined by three parameters (Maidment, 1993).

In this study, hydrological drought was defined using stream flow deficits below the mean as the truncation level. The mean flow was used as the truncation or threshold level since it is more sensitive to extreme values of frequency distributions, it standardizes the severity of maximum and minimum flows and it is indicative of normal hydrological conditions as it lends itself well to revealing the occurrence of hydrological drought (Dracup et al., 1980a and Capra, 1994).

\section{Literature Review}

Hydrological drought frequency modelling involves examining the frequency distribution of best fit for the drought events of duration, magnitude and severity. This is done by statistically fitting several frequency or probability distribution models to the events using either the annual maximum series (AMS) or the partial duration series (PDS) modelling approaches. In the AMS approach, the largest event within a hydrometric year is extracted for frequency analysis while in the PDS approach, all drought events above a given truncation level are considered. This means that the PDS approach provides a more consistent definition of the extreme value region compared to the AMS approach which is problematic when a relatively low truncation level is used, since in some years drought conditions may not occur at all and flow never becomes less than the truncation level. The implication of this is that the AMS approach includes zero values such that when there are too many zero-drought years, the sample size is reduced and the modelling of the drought events is seriously affected. Consequently, the PDS approach is normally used in drought frequency analysis (Zelenhasic and Salvai, 1987; Madsen and Rosbjerg, 1995) and so it was adopted in this study. It includes all the drought events in a time series and therefore intuitively provides a more consistent definition of the extreme value region. It has been used by various researchers including Zelenhasic and Salvai (1987); Madsen and Rosbjerg (1995) and has been recommended by Tallaksen et al. (1997) in hydrological drought frequency studies.

To derive the risk of occurrence related to drought events, the frequency distribution that best describes the past characteristics of the events need to be known. This requires that a suitable drought frequency model be determined from a group of competing ones through fitting the available historical hydrological drought events time series to various frequency distribution models and selecting using a suitable criteria the most appropriate frequency distribution model. Empirically, this involves fitting several statistical frequency distributions to the events and then selecting the model of best fit for the events. The frequency distribution models to be fitted should be those that comply with practical, statistical and physical considerations. Although distributions bounded below by zero and defined by no more than three parameters are normally used in drought events modelling, it is important to note that the existence or otherwise of lower and upper bounds gives no clue as to the true distribution of the underlying variable. This is, for instance, why the Beta distribution although it allows an explicit definition of both lower and upper bounds is never used as, in practice, its upper bound will not be attained without a drastic change in river regime implying that discharge remains zero for the entire year (Clausen and Pearson, 1995).

Drought frequency modelling has received increased attention for a long time despite problems of data availability. The drought events are extracted from the stream flow series using various techniques such as the runs approach, percentile method or the discrete Markov processes (Yevjevich, 1967, 1972) and then fitted to various frequency distributions. By the runs approach, drought events are defined as periods when the hydrological determinant (stream flow) is less than a certain threshold and a single drought event comprises duration and severity or cumulative water deficit. Drought magnitude or the average water deficit is obtained from the ratio of severity to duration. The truncation level is a component by which drought events are abstracted from the remainder of the hydrological time series and divides the series into 'above normal' and 'below normal' sections. It is normally chosen to be some measure of the central tendency of the time series and can either be mean, median or mode. However, the mean is usually preferred since it is more sensitive to the extreme values of frequency distributions, standardizes the severities of high and drought flows and attributes more significance to the extremes of the drought distribution which frequency analysis is most concerned about (Bonacci, 1993).

In modelling drought events, different frequency distributions fitted to various drought events and then the best fitting model identified using chosen criteria. The candidate distributions used for fitting include the Three Parameter Lognormal, Pearson Type III, Extreme Value Type III, Gamma and the Weibull (Normalized Extreme Value) distribution. Others are the Logistic and the Generalized Pareto. Descriptions and applications of these distributions are available in Maidment (1993) and other standard texts on statistical frequency analysis. Modelling of drought events has applications in assessing drought risks, and this has implications to the long 
term ecological and socio-economic well being of the biological and human communities that utilize water in streams (Waylen and Woo, 1987).

The identification of the frequency distribution model of best fit for each of the drought events requires that goodness-of-fit tests be applied. The available tests are grouped into descriptive and predictive ability tests. Descriptive tests are used to find the distribution that best fits the observed sample data and are based on moment ratio diagrams, condition of separation and regional behaviour of statistics (Cunane, 1987). The predictive ability tests are, on the other hand, used to investigate how well a particular distribution together with its associated method of parameter estimation estimates the frequency of the events.

To determine parameters of the various distributions, different estimation techniques may be used. These include graphical, least squares, method of moments, method of maximum likelihood, principle of maximum entropy and those based on probability weighted moments. The graphical, least squares and moments methods are easy and simple to apply but are biased, subjective and suffer from low accuracy and so their usefulness in practical hydrological frequency modelling is quite limited. The method of maximum likelihood is theoretically most suitable in providing unbiased and efficient parameter estimates, but several tests are required to objectively discriminate against various competing frequency distribution models. On the other hand, procedures based on probability-weighted moments (PWMs) are the most modern and are currently widely used in hydrological frequency studies worldwide.

Al-Mashidani et al (1980) fitted several frequency distributions to drought events in the Tigris river and applied the chi-square, Kolmogorov-Smirnov and likelihood ratio tests to discriminate against the normal, lognormal, square root normal, extreme value type I and III and 2-parameter Gamma distributions and found that, except for the chi-square test that accepted all but the normal distribution, the Kolmogorov-Smirnov test rejected none. The extreme value type I, on the other hand, fitted the drought events well using the likelihood ratio test.

Drought is a natural phenomenon whose occurrence cannot be predicted with certainty. Drought events must therefore be treated as random variables to be investigated by the theories of probability and stochastic processes. Knowledge of frequency distribution of drought duration and severity is vital in the performance of any water resources system (Sen, 1980). Using different truncation levels to define monthly and seasonal drought occurrences, Woo and Tarhule (1994) fitted the normal, exponential, and Weibull frequency distributions to drought duration, deficit and time of occurrence in four Nigerian streams and selected the distribution of best fit using small root-mean-square error values, Kolmogorov-Smirnov test and theoretical considerations. The results showed the duration of short droughts to be best described by the Weibull distribution model and the duration and starting dates of drought to be normally distributed. Later, Madsen and Rosbjerg (1995) in a frequency analysis study of drought duration and deficit in two Danish streams evaluated the suitability of several distributions in modelling drought events and found drought duration to fit the normal distribution while the General Pareto Distribution fitted drought severity. Elsewhere, Rossi et al. (1992) have provided a detailed review of the existing methodologies for estimating and analysing regional droughts and their spatial and temporal variability. Another notable hydrological drought modelling study is that of Clausen and Pearson (1995).

In Kenya, hydrological drought frequency studies are few and scattered and except for work by Mutua (1993) and Opere (1999) who fitted several distributions to maximum flow in the Lake Victoria, Rift Valley, Athi and the Tana basins and found the Wakeby and the three-parameter lognormal distributions to be the best models for flood frequency analysis, no comprehensive study has been done to fit frequency distribution models to hydrological drought events. In examining hydrological drought events using stream flow records, it is necessary to differentiate droughts from low flows. Low flows differ from droughts in that the duration in low flows is of the order of days or weeks, flows occur instantaneously and only one event is selected from an annual period of data. The various frequency distributions used by Mutua (1993) and Opere (1999) in modelling floods in the country are useful in hydrological drought frequency analysis. However, their suitability or otherwise has not been examined and except for preliminary studies by Sharma (1996) who has shown drought duration and severity at selected gauging stations in the country to follow the three parameter lognormal distribution, no previous attempt has been made to test the suitability of various frequency distribution models in modelling drought duration and severity in the Tana basin.

\subsection{Data analysis methods}

Frequency distributions contain parameters that need to be estimated before being used as simulating tools for drought events. These parameters are mathematical indices characteristic of a statistical distribution, have a physical significance and can completely define the mathematical properties of a distribution. Several approaches of estimating the parameters of the distributions include the method of moments, maximum likelihood, least squares, graphical, probability-weighted moments (PWMs) and the L-moments technique. The first four techniques are relatively simple and easy to apply but they provide parameters that are biased and subject to large 
sampling errors. The L-moments procedure (Hosking and Wallis, 1993) was applied in the study since it provides unbiased parameters that are not subject to large sampling errors.

L-moments are defined to be linear combinations of probability-weighted moments (PWMs) and together are analogous to ordinary moments since they summarize theoretical probability distributions of observed samples. These moments can also be used for parameter estimation, interval estimation and hypothesis testing and can be estimated using linear combinations of an ordered data set. Further, sample estimators of Lmoments, which are linear combinations of ranked observations, do not involve squaring or cubing of observations. Consequently, estimators based on these moments are subject to less bias and have very nearly a normal distribution while those based on ordinary product-moments requires squaring and cubing of observations causing them to give greater weights to the observations far from the mean resulting in substantial bias and variation in small samples (Potter and Lettenmaier, 1990).

The L-moment estimators also provide more accurate quantile estimators than product moment and maximum likelihood estimators, they characterize a wider range of distributions, are less biased to estimation, are more robust to the presence of outliers in the data, approximate their asymptotic normal distribution more closely in finite samples and frequently yield accurate estimation of parameters of fitted distributions (Vogel et al, 1993). Because of these reasons, the L-moments technique has lately found wide application in hydrological frequency analysis studies in various parts of the world and was therefore found to be ideal for use in this study. The simplest way of defining L-moments is by using probability-weighted moments since L-moments are linear combinations of PWMs. Probability weighted moments (PWMs) may be defined (Greenwood, et al., 1979) using equation (1).

$\beta_{\mathrm{r}}=\mathrm{E}\left\{\mathrm{X}\left[\mathrm{F}_{\mathrm{X}}(\mathrm{x})\right]^{\mathrm{r}}\right\}$

Where,

$\beta_{\mathrm{r}}$ is the $\mathrm{r}^{\text {th }}$ order PWM and $\mathrm{F}_{\mathrm{X}}(\mathrm{x})$ is the cumulative distribution function of $\mathrm{X}$.

When $r=0, \beta_{0}$ is the mean stream flow and so a sample estimate of the first PWM denoted by $b_{0}$ is the sample mean.

The first four unbiased sample estimates of the PWMs for any distribution can be computed using equations (2) to equation (5).

$$
\begin{aligned}
& \beta_{0}=\mathrm{m}=1 / \mathrm{n} \sum_{j=1}^{n} \mathrm{x}_{\mathrm{j}} \\
& \beta_{1}=\sum_{j=1}^{n-1}\left[(\mathrm{n}-\mathrm{j}) /(\mathrm{n}(\mathrm{n}-1)] \mathrm{x}_{\mathrm{j}}\right. \\
& \beta_{2}=\sum_{j=1}^{n-2}[(\mathrm{n}-\mathrm{j})(\mathrm{n}-\mathrm{j}-1) / \mathrm{n}(\mathrm{n}-1)(\mathrm{n}-2)] \mathrm{x}_{\mathrm{j}} \\
& \beta_{3}=\sum_{j=1}^{n-3}[(\mathrm{n}-\mathrm{j})(\mathrm{n}-\mathrm{j}-1)(\mathrm{n}-\mathrm{j}-2) / \mathrm{n}(\mathrm{n}-1)(\mathrm{n}-\mathrm{j}-2)] \mathrm{x}_{\mathrm{j}}
\end{aligned}
$$

Where,

$\mathrm{x}_{(\mathrm{j})}$ represents the ordered statistics.

From the expressions in the above equations, the first four L-moments are obtained for the population using the expressions in equations (6) to (12) while those for the sample can be obtained by substituting $b_{1}, b_{2}, b_{3}, b_{0}$ in the equations.

$$
\begin{aligned}
& \lambda_{1}=\beta_{0} \\
& \lambda_{2}=\beta_{0}-2 \beta_{1} \\
& \lambda_{3}=\beta_{0}-6 \beta_{1}+6 \beta_{2} \\
& \lambda_{4}=\beta_{0}-12 \beta_{1}+30 \beta_{2}-20 \beta_{3} \\
& \tau_{2}=\lambda_{2} / \lambda_{1} \\
& \tau_{3}=\lambda_{3} / \lambda_{2} \\
& \tau_{4}=\lambda_{4} / \lambda_{3}
\end{aligned}
$$




\section{Selection of best fitting frequency distribution}

The distributions of best fit for the drought events were identified using the $\mathrm{Z}$ value obtained from the average L-moment statistics of a particular candidate distribution and the average $L$-moment statistics. The $Z$ value for each homogenous region was determined from sample estimates of $\mathrm{L}_{\mathrm{cv}}, \mathrm{L}_{\mathrm{cs}}$ and $\mathrm{L}_{\mathrm{ck}}$ that were determined from probability weighted moment estimators and the weighted means of $\mathrm{L}_{\mathrm{cv}}, \mathrm{L}_{\mathrm{cs}}$ and $\mathrm{L}_{\mathrm{ck}}$ using records from the river gauging stations representing each region. Using the P3, GEV, GL and GN distributions, random samples were generated and $\mathrm{L}_{\mathrm{cv}}, \mathrm{L}_{\mathrm{cs}}$ and $\mathrm{L}_{\mathrm{ck}}$ estimated for each simulated sequence and the average value for the sequence obtained together with the overall average value and the standard deviation of the mean values from the simulations done. A frequency distribution was selected as the best fitting if $\left|Z^{\text {Dis }}\right| \leq 1.64$. Although several distributions passed the goodness-of-fit criteria, the one with the lowest $\left|Z^{\text {Dis }}\right|$ value was taken as the distribution of best fit. The goodness-of-fit measure, $Z^{\text {Dist }}$ was then determined using equation (13).

$\mathrm{Z}^{\text {Dist }}=\left(\tau_{4}{ }^{\text {Dist }}-\overline{\tau_{4}}+\beta_{4}\right) / \sigma_{4}$

Where,

$\tau_{4}^{\text {Dist }}$ is the average L-kurtosis from data of a given site, $\overline{\tau_{4}}$ is the average L-kurtosis from simulation for a fitted distribution, $\beta_{4}$ and $\sigma_{4}$ are the bias and the standard deviation of $\overline{\tau_{4}}$, respectively.

\section{Results and Discussion}

The frequency analysis results for drought duration are shown in Table 1 for the four homogeneous regions. The model of best fit in each homogeneous region was selected using the Z-statistic goodness of fit whose values are also given in the Table. The statistic was used to judge how well the regionally averaged Lstatistics values matched the simulated ones in the homogeneous regions.

Table 1: Regional frequency model for drought duration

\begin{tabular}{|l|l|l|}
\hline Region & Frequency distribution model & Z-value \\
\hline R1 & Generalized Normal & 0.87 \\
& Generalized Pareto & 1.37 \\
& Pearson Type III & -2.42 \\
& Generalized Extreme Value & 2.88 \\
& Generalized Logistic & 3.03 \\
\hline R2 & Generalized Extreme Value & 0.88 \\
& Generalized Pareto & 1.37 \\
& Generalized Normal & 1.62 \\
& Generalized Logistic & 2.03 \\
& Pearson Type III & -2.32 \\
\hline R3 & Generalized Normal & 0.67 \\
& Generalized Extreme Value & 0.86 \\
& Generalized Logistic & 3.03 \\
& Generalized Pareto & 2.07 \\
& Pearson Type III & -2.12 \\
\hline R4 & Generalized Normal & 0.97 \\
& Generalized Logistic & 1.03 \\
& Generalized Pareto & 1.77 \\
& Generalized Extreme Value & 1.88 \\
& Pearson Type III & -2.62 \\
\hline
\end{tabular}

The model of best fit is one for which $\mathrm{Z} \leq 1.64$. From the Table, drought duration in Region 1 (R1) is best fitted by the Generalized Normal (GN) distribution followed by the Generalized Pareto (GPA), Pearson Type III (P3), Generalized Extreme Value (GEV) and General Logistic (GL), in that order. The respective absolute Z-statistic values for these distributions are $0.87,1.37,2.42,2.88$ and 3.03 which clearly shows the model of best fit for drought duration in this region is the Generalized Normal (GN) or the 3-Parameter Lognormal whilst the model of worst fit is the Generalized Logistic (GL). For Region 2 (R2), the model of best fit was the GEV with a Z-statistic value of 0.88 followed by the GPA whose Z-value was 1.37 . The worst model in this region was the Pearson type III (P3) with a Z-statistic value of 2.32. The order of preference of the frequency distribution models in Region 2 (R2) is Generalized Extreme Value (GEV), Generalized Pareto (GPA), Generalized Normal (GN), Generalized Logistic (GL) and Pearson Type III (P3) with Z-values of 0.88, $1.37,1.62,2.03$ and 2.32, respectively. For Region 3 (R3), the best fitting distribution was the GN whilst the worst fitting frequency distribution was the GL with the order of model preference being GN, GEV, GPA, P3 and GL with corresponding Z-values of $0.67,0.86,2.07,2.12$ and 3.03 , respectively. The order of model 
preference in Region 4 (R4) from the best to the worst fitting was GN, GL, GPA, GEV and P3 with respective Z-statistic values of $0.97,1.03,1.77,1.88$ and 2.62. The Generalized Normal was the overall best model for fitting drought duration in the basin as it was best in Regions 1, 3 and 4 (R1, R3 and R4) whilst the model of worst fit was the GL in region 1 and region 3 (R1 and R3) but that in regions 2 and 4 (R2 and R4) was P3.

The frequency modelling result for severity is shown in Table 2 . The findings show the model preference in R1 to be GN, GPA, P3, GEV and GL with respective Z-statistic values of $0.82,0.91,0.96,1.29$ and 3.82. The worst performing frequency distribution model in R2, R3 and R4 was found to be P3 except for R1 where the model of worst fit was the GL. The overall model of best fit for severity was the Generalized Normal as it provided the frequency distribution model of best fit in three of the four regions in the basin. The model of worst fit for severity was P3 in three of the four regions. The order of preference in the performance of the various fitted frequency models is shown in Table 3. The findings in this study are consistent with those of Al-Mashidani et al (1980), Srikanthan and McMahon (1985), Woo and Tarhule (1994); Madsen and Rosbjerg (1995); Sharma (1996), Tallaksen and Hisdal (1997). The GEV distribution is ideally the model of best fit for drought duration and severity as it is theoretically the parent distribution of extreme drought flows. However, it performed poorly in this study probably because it requires a relatively complex numerical algorithm not suitable for many practical applications (Vogel and Kroll, 1989). It has, nevertheless, been found suitable in modelling flood events and to provide flood flow estimates in most Kenyan catchments by Opere (1999).

From the frequency results in Table 3, the GN distribution is the best fitting frequency distribution for drought duration and severity. But, the P3 distribution did not perform well since it requires at site estimates of skew coefficients that are not precise for the small samples typically encountered in practice and is normally used in modelling flood flow events. The estimated parameters for the generalized normal distribution are presented in Table 5 and the cumulative distribution function $\mathrm{F}(\mathrm{x})$ is given in equation (14).

$$
\mathrm{F}(\mathrm{x})=\Phi\{-\log [1-k(x-u) / a] / k\}
$$

Where $\Phi$ is the standard normal cumulative function; $\mathrm{u}, \mathrm{a}$ and $\mathrm{k}$ are parameters describing location, scale and shape of the distribution.

Table 3: Regional frequency model for drought severity

\begin{tabular}{|l|l|l|}
\hline Region & Type of frequency distribution & Z-value \\
\hline R1 & Generalized Normal, GN & -0.82 \\
& Generalized Pareto, GP & -0.91 \\
& Pearson Type III, P3 & 0.96 \\
& Generalized Extreme Value, GEV & 1.29 \\
& Generalized Logistic, GL & -3.82 \\
\hline R2 & Generalized Extreme Value & -0.81 \\
& Generalized Pareto & -0.92 \\
& Generalized Normal & 0.96 \\
& Generalized Logistic & 1.39 \\
& Pearson Type III & -3.62 \\
\hline R3 & Generalized Normal & -0.72 \\
& Generalized Extreme Value & -0.81 \\
& Generalized Logistic & 0.86 \\
& Generalized Pareto & 1.19 \\
& Pearson Type III & -3.52 \\
\hline R4 & Generalized Normal & -0.80 \\
& Generalized Logistic & -0.91 \\
& Generalized Pareto & 0.94 \\
& Generalized Extreme Value & 1.24 \\
& Pearson Type III & -3.22 \\
\hline
\end{tabular}

Table 4: Regional parameters for drought duration and severity

(a) Drought duration

\begin{tabular}{|l|l|l|l|}
\hline Region & U & A & K \\
\hline R1 & 0.54 & 0.46 & -0.44 \\
R2 & 0.52 & 0.45 & -0.43 \\
R3 & 0.48 & 0.40 & -0.42 \\
R4 & 0.52 & 0.44 & -0.41 \\
\hline
\end{tabular}

(b) Drought severity

\begin{tabular}{|l|l|l|l|}
\hline Region & $\mathrm{u}$ & $\mathrm{A}$ & $\mathrm{K}$ \\
\hline R1 & 0.56 & 0.47 & -0.42 \\
R2 & 0.55 & 0.48 & -0.46 \\
R3 & 0.53 & 0.41 & -0.43 \\
R4 & 0.54 & 0.45 & -0.44 \\
\hline
\end{tabular}


The parameters in Table 4 can be used to compute standardized quantile estimates for different return periods for both duration and severity in the homogenous regions of the basin. From the parameters obtained, dimensionless quantiles for duration and severity in the four homogeneous regions of the basin can then be derived, which together with respective means of the events, can be used to provide estimates of both duration and severity at specified return periods in each of the regions. From the expected magnitudes of the drought events at different return periods, planning of water dependent activities can be done to ensure availability of water for various applications.

\section{Conclusion and Recommendations}

The overall frequency distribution of best fit for drought duration and severity was found to be the Generalized Normal whilst the Pearson Type III frequency distribution was the distribution of worst fit for both drought duration and severity in the basin. Some of the recommendations following the findings of the study include

a) The need for public education and the need to institute various mechanisms of maintaining holdover storages, lowering intakes to groundwater supplies and adopting various water conservation activities such as reforestation, basin and catchment rehabilitation within the various zones of the basin.

b) Promotion of strip cropping, terracing, storage of water in farm ponds and back of check dams and reservoirs to provide sufficient water for domestic, municipal, hydropower, agricultural and industrial activities in the basin. This means that available water resources in the basin should be managed in an integrated manner to ensure sufficient availability of water to maintain supplies for different uses.

c) Limiting and/or adjusting different economic development activities within the basin to available water. The various zones prone to drought occurrences should be used to guide future development policies and endeavours in this regard.

d) Instituting a comprehensive policy and legislation on drought management in areas where drought is recurrent. This should form the basis for integration of drought management issues into national and local development programmes. A suitable mechanism should be found of bringing together all the stakeholders such as government, nongovernmental and international organizations, Institutions of higher learning, research institutes and the local communities to be actively involved in various aspects of drought management in the basin. An early warning and national monitoring system will also need to be established to ensure continuous assessment of drought impact in various regions of the basin.

\section{Acknowledgements}

This study was done based on the financial support of the German government through the DAAD Kenya office. The financial assistance is greatly appreciated. The late Prof. Wellington Wamicha was very instrumental in the conceptualization and completion of the study.

\section{References}

[1] Al-Mashidani, G., Pande, B. B. L. and Qadri, I. 1980. Drought flow Analysis of the River Tigris at Baghdad. Hydrol. Sci. Bull., 25 (4): 453-459.

[2] Bonacci, O. 1993. Hydrological Identification of Drought. Hydrological Processes, 7: 249-262.

[3] Capra, A. 1994. Application of Fuzzy sets to Drought classification. Advances in Water Resources Technology and Management. Balkema, Rotterdam. PP 479-483.

[4] Chowdhury, J. U., Stedinger, J. R. and Lu, L. H. 1991. Goodness of fit tests for Regional Generalized Extreme Value Flood Distribution. Water Resources Research 27(7): 1765-1777.

[5] Clausen, B. and Pearson, P. 1995. Regional Frequency Analysis of Annual Maximum Streamflow Drought. Journal of Hydrology, 173: $111-130$

[6] Cunane, C. 1987. Review of Statistical Models for Flood Frequency Estimation. In: Singh, V. P., (Editor), Hydrologic Frequency Modeling, Reidel, Dordrecht. PP 49-95.

[7] Dracup, J. A., Lee, K. S. and Paulson, E. G., Jr. 1980. On the Definition of Droughts. Water Resources Research, 16 (2): $297-302$

[8] Greenwood, J. A., Landwehr, J. M., Matalas, N. C. and Wallis, J. R. 1979. Probability Weighted Moments: Definitions and Relation to Parameters of Distributions Expressible in Inverse Form. Water Resources Research 15 (5): 1046-1054.

[9] Hosking, J. R. M. and Wallis, J. R. 1993. Some Statistics useful in Regional Frequency Analysis. Water Resources Research, 29 (2): 271-281.

[10] Madsen, H. and Rosbjerg, D. 1995. On the Modeling of Extreme Droughts. Proceedings of an International Symposium on Modelling and Management of Sustainable Basin Scale Water Resource Systems, Boulder, Colorado. IAHS Publication No. 231. PP 377- 385.

[11] Maidment, D. R. 1993. Handbook of Hydrology. McGraw-Hill, New York, USA.

[12] Mutua, F. M. 1993. The Use of Akaike Information Criterion in the Identification of Optimum Flood Frequency Model. Proceedings of the First International Conference of the African Meteorological Society, Nairobi, Kenya. PP 871-881.

[13] Opere, A. O. 1999. Space-Time Characteristics of Stream flow in Kenya. Unpublished PhD Thesis, University of Nairobi.

[14] Potter, K. W. and Lettenmaier, D. P. 1990. A Comparison of Regional Flood Frequency Estimation Methods Using a Resampling Method. Water Resources. Research. 26(3): 415-424.

[15] Rossi, G., Benedini, M., Tsakiris, G. and Giakoumakis, S. 1992. On Regional Drought Estimation and Analysis. Water Resources Management, 6: 249-277.

[16] Sen, Z. 1980. Regional Drought and Flood Frequency Analysis: Mathematical Considerations. Journal of Hydrology, 46: 265-279. 
[17] Sharma, T. C. 1996. Prediction of Extremal Droughts. Proceedings of the International Conference on Water Resources and Environmental Research, Vol. I, Kyoto, Japan. PP 515-522.

[18] Smakhtin, V. U. 2001. Low flow Hydrology: A Review. Journal of Hydrology, 240 (2001), 147-186.

[19] Srikanthan, R. and McMahon, T. A. 1985. Recurrence Interval of Drought Events through Stochastic Analysis of Rainfall and Stream Flow Data. Hydrological Sciences Journal, 30 (2):197-205.

[20] Tallaksen, L. M., Madsen, H. and Clausen, B. 1997. On the Definition and Modeling of Drought Duration and Deficit Volume. Hydrological Sciences Journal, 42 (1): 15 - 33.

[21] Vogel, R. M. and Kroll, C. N. 1989. Low Frequency Analysis using Probability Plot Correlation Coefficients. Journal of Water Resources Planning and Management, ASCE, 115 (3): 338-357.

[22] Waylen, P. R. and Woo, M. K. 1987. Annual Low Flows Generated by Mixed Processes. Hydrological Sciences Journal, 32(3): 371-383.

[23] oo, M. K. and Tarhule, A. 1994. Stream Flow Droughts of Northern Nigerian Rivers. Hydrological Sciences Journal, 39 (1): $19-33$. 